\title{
Intramyocardial injection of tannic acid attenuates postinfarction remodeling: A novel approach to stabilize the breaking extracellular matrix
}

Hao Zhang, MD, PhD, ${ }^{\text {a,b }}$ Shen-jun Zhu, MD, ${ }^{a}$ De Wang, MD, PhD, ${ }^{a}$ Ying-jie Wei, PhD, ${ }^{\text {a }}$ and Sheng-Shou Hu, MD ${ }^{\text {a,b }}$

\begin{abstract}
Objective: Myocardial infarction is associated with early matrix metalloproteinase activation and extracellular matrix degradation. We tested the hypothesis that stabilizing the original extracellular matrix of the infarcted left ventricle with local injection of tannic acid would preserve cardiac structure and function.
\end{abstract}

\begin{abstract}
Methods: In vitro cytotoxicity of tannic acid was performed first; myocardial infarction model was induced by ligation of the left anterior descending branch in rats. Tannic acid was intramyocardially injected into infarcted site 24 hours after myocardial infarction $(n=30)$, and saline solution was injected in the same way as in the control $(\mathrm{n}=30)$. The matrix metalloproteinase activity from tannic acid/saline solution-treated tissues was assayed by gelatin zymography 24 hours and 1 week after the treatment. The collagen content in the infarcted area was evaluated by hydroxyproline colorimetry assay 1 and 4 weeks after the treatment. Left ventricular structure and function were also evaluated with echocardiography, hemodynamics, and histologic examination.
\end{abstract}

Results: Tannic acid at a concentration of $0.05 \%$ had minimal cytotoxic effects on cultured cardiomyocytes and thus was subsequently chosen as the optimal concentration for injection. Compared with the saline solution injection group, tannic acid treatment inhibited the matrix metalloproteinase-2/-9 activity and increased the collagen content at the early post-myocardial infarction stage ( $48.6 \pm 7.2 \mathrm{vs} 37.3 \pm 6 \mu \mathrm{g} / \mathrm{mg}$ dry weight). Tannic acid treatment also significantly reduced infarct expansion (infarct expansion index: $1.04 \pm 0.15$ vs $1.42 \pm 0.21$ ) and left ventricular dilatation at 4 weeks after infarction. Although tannic acid treatment improved fractional shortening ( $26 \% \pm 2.4 \%$ vs $23.3 \% \pm 3.2 \%$ ), it failed to alter blood pressure (systolic blood pressure: 93.8 $\pm 8.2 \mathrm{vs} 90.6 \pm 8.5 \mathrm{~mm} \mathrm{Hg}$ ) and rate of pressure rise.

Conclusions: Local delivery of tannic acid prevents collagen matrix degradation via cross-linking fibrous collagen and inhibiting matrix metalloproteinase activity but does not improve the intrinsic contractile function of myocardium. This treatment may be helpful to attenuate the adverse topographic remodeling after acute myocardial infarction.

\section{Supplemental material is available online.}

Cardiac extracellular matrix (ECM) mainly consists of a 3dimensional collagen architecture, which is critical to retain tissue integrity and facilitate the conversion of single cardiocyte contraction into global pump function. In the setting of myocardial infarction (MI), the activation of matrix metalloproteinase (MMP) and enzymatic degradation of ECM play critical roles in scar expansion, ventricular dilation, ventric-

From the Research Center for Cardiac Regenerative Medicine, the Ministry of Health, ${ }^{\mathrm{a}}$ and Department of Cardiac Surgery, ${ }^{\mathrm{b}}$ Cardiovascular Institute and Fu Wai Hospital, Chinese Academy of Medical Science and Peking Union Medical College, Beijing, China.

This research was supported by grants from Program for Changjiang Scholars and Innovative Research Team in University (S.H.), Natural Science Foundation of China (30500498) (H.Z.), and Beijing Nova Project (2006A85) (H.Z.).

Received for publication Jan 18, 2008; revisions received June 10, 2008; accepted for publication July 6, 2008.

Address for reprints: Sheng-Shou Hu, MD, Cardiovascular Institute and Fu Wai Hospital, Chinese Academy of Medical Science, Department of Cardiac Surgery, Beilishilu Rd 167A, Beijing 100037, P.R. China (E-mail: shengshouhu@yahoo.com). J Thorac Cardiovasc Surg 2009; 137:216-22

$0022-5223 / \$ 36.00$

Copyright (c) 2009 by The American Association for Thoracic Surgery doi: 10.1016/j.jtcvs.2008.07.030 ular aneurysm formation, and even cardiac rupture. Early infarct expansion initiates adverse remodeling, leads to left ventricular dilation, and portends a poor long-term outcome. To retard the early adverse post-MI remodeling, various efforts have been attempted to preserve or restore the ECM after MI (discussed in details in the excellent review by Spinale ${ }^{1}$ ).

Tannic acid (TA), a natural extract of plant polyphenol, has been shown to physically cross-link collagen and elastin and hence improve their resistance to the enzymatic degradation. ${ }^{2,3}$ Collagen is a protein with a fiber structure and, by introducing multiple hydrogen bonds, can be physically cross-linked by TA and resist to resolution and enzymatic degradation. Furthermore, in previous tumor studies, it has been suggested that TA can inhibit MMP activities and thus suppress the invasiveness and metastasis of cancer cells. ${ }^{4}$

Considering the above facts, we hypothesized that intramyocardial injection of TA at the early stage of MI would stabilize collagen matrix and attenuate early left ventricular $(\mathrm{LV})$ remodeling via direct collagen cross-linking and MMP activities suppression. Therefore, in this study we wanted to address: (1) the biosafety of TA for in vivo use; (2) whether local injection of TA can maintain the integrity of ECM architecture and thus thicken the scar tissue and limit infarct 


$\begin{aligned} & \text { Abbreviations and Acronyms } \\ & \text { DMEM }=\text { Dulbecco's modified Eagle medium } \\ & \text { ECM }=\text { extracellular matrix } \\ & \text { EDD }=\text { end-diastolic dimensions } \\ & \text { ESD }=\text { end-systolic dimensions } \\ & \text { FS }=\text { fractional shortening } \\ & \text { LV }=\text { left ventricular } \\ & \text { MI }=\text { myocardial infarction } \\ & \text { MMP }=\text { matrix metalloproteinase } \\ & \text { MTT }=3 \text {-[4,5-dimethylthiazol-2-yl]-2, } \\ & \text { 5-diphenyl tetrazolium bromide } \\ & \text { OCT }=\text { optimum cutting temperature } \\ & \text { PBS }=\text { phosphate-buffered saline solution } \\ & \text { SD }=\text { Sprague-Dawley } \\ & \text { TA }=\text { tannic acid } \\ & \text { TIMP-2 }=\text { tissue inhibitor of metalloproteinase-2 }\end{aligned}$

expansion; (3) whether TA injection can improve damaged heart function; and (4) the underlying mechanisms behind TA treatment.

\section{METHODS}

\section{In vitro Cytotoxicity Assessment}

Cardiomyocytes were obtained by enzymatic digestion of the left ventricles from Sprague-Dawley (SD) rats, as previously described. ${ }^{5}$ The cells were seeded in 96-well plates (5000 cells/well) in Dulbecco's modified Eagle medium (DMEM) with $10 \%$ fetal bovine serum, in a humidified incubator at $37^{\circ} \mathrm{C}$ with $5 \% \mathrm{CO}_{2}$. When the cells were $95 \%$ confluent, they were exposed to increasing concentrations of TA $(0.025 \%, 0.05 \%$, $0.10 \%, 0.15 \%$, and $0.20 \%$ TA in phosphate-buffered saline solution [PBS]), and cell viability was assessed by the MTT (3-[4,5-dimethylthiazol-2-yl]-2,5-diphenyl tetrazolium bromide) assay as previously described. ${ }^{6}$ Briefly, solution of MTT (Sigma-Aldrich, St Louis, Mo) was prepared in DMEM and added to each well at a final concentration of $0.5 \mathrm{mg} / \mathrm{mL}$ after removal of the culture medium, after which the cells were incubated at $37^{\circ} \mathrm{C}$ for 4 hours. The supernatants were removed from the wells, and $100 \mu \mathrm{L} \mathrm{di-}$ methyl sulfoxide was added to each well to dissolve the blue formazan product. Quantitative colorimetric assay at $570 \mathrm{~nm}$ was measured on a 96-well scanning spectrophotometer. Cardiomyocytes were also incubated with PBS and $70 \%$ ethanol as negative and positive controls, respectively.

\section{Animal Experiments}

Acute myocardial infarction was induced by ligation of the proximal left coronary artery in 8-week-old male SD rats (280 to $300 \mathrm{~g}$ ) as previously described. ${ }^{7}$ All animal experimental procedures were approved by the Ethic Committee for Animal Study in Fuwai Hospital and conformed to the guidelines in the "Regulation to the Care and Use of Experimental Animal" (1996) of the Beijing Council on Animal Care. In brief, the animals were intubated with an 18-gauge intravenous catheter and ventilated at a rate of 65 cycles/min with a tidal volume of $2.5 \mathrm{~mL}$ under room air supplemented with oxygen $(0.2 \mathrm{~L} / \mathrm{min})$ and $1.0 \%$ to $2.0 \%$ isoflurane using a Harvard ventilator (model 683, Harvard Apparatus, Holliston, Mass). After the chest was cleaned and shaved, through a left lateral thoracotomy, the proximal left coronary artery was ligated with a $6-0$ polypropylene suture. After myo- cardial ischemia was confirmed by regional myocardial color change, the incision was closed in layers with 3-0 silk running sutures. Twenty-four hours later, the rats were reanesthetized and underwent echocardiography examination. Sixty of the survival rats with LV fractional shortening (FS) at a range of $20 \%$ to $30 \%$ were randomized into 2 groups: TA group $(\mathrm{n}=$ $30)$ and control group $(n=30)$. Then the chest was reopened via the old incision, and the grayish infarcted myocardium was exposed. Then, $100 \mu \mathrm{L}$ of $0.05 \%$ TA or saline solution was injected into 2 sites located in the infarcted and borderline area with a 29 -gauge insulin syringe ( $50 \mu \mathrm{L}$ per site). After the second surgery, the rats were allowed to recover and were housed in a clean laboratory room with a temperature-controlled environment under a 12-hour light-dark cycle and fed with free access to food and water. The entire animal experimental protocol was described in Figure 1.

\section{Assay of MMP/Tissue Inhibitor of Metalloproteinase Expression and Activities}

To assess the inhibitory effect of TA on post-MI MMP activity, 4 rats from each group were killed either 24 hours or 1 week after TA/saline injection. The infarcted LV tissue (injection site) was separated and homogenized. The activity of myocardial collagenases (MMP-2 and -9) was analyzed by gelatin zymography as described elsewhere. ${ }^{8}$ In brief, $8 \%$ substrate gel was prepared with gelatin (final concentration of $1.0 \mathrm{mg} / \mathrm{mL}$ ). A total of $20 \mu \mathrm{g}$ protein was loaded per lane without reduction. After electrophoresis, the gel was washed twice for 15 minutes each with $2.5 \%$ Triton $\mathrm{X}$ 100. Then the gel was incubated overnight at $37^{\circ} \mathrm{C}$ in substrate buffer $(0.5$ $\mathrm{mol} / \mathrm{L}$ Tris- $\mathrm{HCl}, \mathrm{pH} 6.8,0.5 \% \mathrm{CaCl}_{2}, 0.2 \mathrm{~mol} / \mathrm{L} \mathrm{NaCl}, 0.2 \%$ Brij- 35 and $0.02 \% \mathrm{NAN}_{3}$ ). After incubation, the gel was stained for 1 hour in $0.25 \%$ Coomassie brilliant blue R-250 in acetic acid:methanol:water (1:4.5:4.5 by volume), destained in $10 \%$ acetic acid, and scanned for lytic activity and photographed. Gelatinolytic activity of MMP-2 and -9 was detected as transparent bands on the Coomassie brilliant blue-stained gel. To investigate whether the TA can directly inhibit the gelatinolytic activity, TA was added into the incubation buffer with a final concentration of $50 \mu \mathrm{g} / \mathrm{mL}$ in a separate zymography assay with the samples from the control group.

Furthermore, to figure out whether TA can influent the expression of MMP, another 4 rats from each group were killed at 24 hours, and Western blotting was performed to determine the protein level of MMP-2, -9, and one of their endogenous inhibitors, tissue inhibitor of metalloproteinase-2 (TIMP-2), using the corresponding primary antibodies (anti-MMP-2, from Santa Cruz Biotechnology [Santa Cruz, Calif]; anti-MMP-9 and anti-TIMP-2, from Chemicon International, Temecula, Calif).

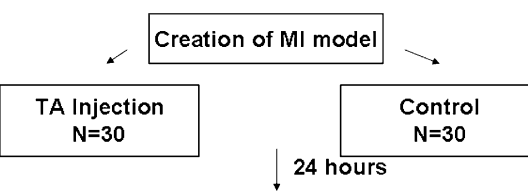

Assay of MMP Activity and Expression $\mathrm{N}=8$ per group

$\downarrow 1$ week

Assay of MMP Activity ( $n=4$ per group) Collagen Content Evaluation ( $n=5$ per group)

$\downarrow \quad 4$ week

Echocardiographic study ( $\mathrm{n}=11$ per group)

Collagen Content Evaluation ( $n=5$ per group)

Invasive Homodynamic Study and Histological Examination ( $n=6$ per group)

FIGURE 1. Scheme of in vivo experimental protocol. $M I$, Myocardial infarction; $T A$, tannic acid; $M M P$, matrix metalloproteinase. 


\section{Collagen Content Evaluation by Hydroxyproline Assay}

One and 4 weeks after TA/saline solution injection, myocardial collagen content in the infarcted region was measured by hydroxyproline assay using a modified Stegemann protocol. ${ }^{9}$ Briefly, samples of infarct myocardium from 5 rats at different times from each group were carefully separated and lyophilized to a constant weight. Each sample was homogenized in $0.1 \mathrm{~mol} / \mathrm{L} \mathrm{NaCl}$ and $5 \mathrm{mmol} / \mathrm{L} \mathrm{NaHCO}_{3}$, washed with the same solution 5 times, and hydrolyzed in $1 \mathrm{~mL}$ of $6 \mathrm{~mol} / \mathrm{L} \mathrm{HCl}$ for 24 hours at $100^{\circ} \mathrm{C}$, followed by neutralization with $6 \mathrm{~mol} / \mathrm{L} \mathrm{NaOH}$. Isopropanol, chloramine- $\mathrm{T}$ reagent, and Ehrlich's reagent solution were added, and the samples were mixed and incubated at $60^{\circ} \mathrm{C}$ for 30 minutes. The absorbency of the samples was read at $560 \mathrm{~nm}$, and the hydroxyproline concentration was assessed using a curve obtained from hydroxyproline standard solutions. Data were expressed as micrograms of collagen per milligram dry weight, assuming that collagen contains an average of $13.5 \%$ hydroxyproline.

\section{Postmortem Histologic Examination}

After echocardiography and hemodynamic examination, 6 rats of each group were deeply anesthetized and their hearts were exposed through a median sternotomy. After injection of $10 \%$ potassium chloride to arrest the heart in diastole, the heart was harvested, washed, and immersed in $4 \%$ paraformaldehyde for at least 24 hours. The fixed hearts were equidistantly cut into 3 transverse slices. The middle slice was embedded in paraffin and then sectioned at $5 \mu \mathrm{m}$ for picrosirius red staining. Histologic imagines of the stained sections were photographed with Olympus DP70 (Olympus Optical Co, Ltd, Tokyo, Japan) and analyzed with Image-Pro Plus 6.0 Software (Media Cybernetics, Inc., Bethesda, MD). The following parameters were measured: (1) the thickness of scar and septum (average of 5 equidistant measurements); (2) epicardial and endocardial circumference, composed by scar and noninfarcted tissue, respectively; (3) areas composed by collagen and noninfarcted muscle, area of LV cavity, and area of total LV. To evaluate the degree of LV dilation, the expansion index was calculated as LV cavity area/total LV area $\times$ septum thickness/scar thickness, as defined by Hochman and Choo. ${ }^{10}$ All histologic procedure and assay were performed in a blinded fashion.

To demonstrate the ECM microstructure, LV tissue was also stained as the method described by Rhaleb and colleagues. ${ }^{11}$ Briefly, the fresh samples were embedded in optimum cutting temperature (OCT) compound and cryosectioned at $5 \mu \mathrm{m}$. Then the sections were treated with $3.3 \mathrm{U} /$ $\mathrm{mL}$ neuroaminidase type $\mathrm{V}$ (Sigma-Aldrich) for 1 hour at room temperature. After washing in PBS, the sections were stained with $0.10 \mathrm{mg} / \mathrm{mL}$ fluorescein-labeled peanut agglutinin (Vector Laboratories, Burlingame, Calif). The fields at borderline zone were photographed.

\section{Cardiac Structure and Function Assessment}

LV dimension and function were assessed by 2-dimensional echocardiography in isoflurane-anesthetized animals perioperatively (pre-TA injection and 2 weeks after TA/saline injection. Although echocardiographic examination was performed for all surviving rats, only 11 rats from each group received echo assessments at the experimental end point ( 4 weeks after treatment). Transthoracic echocardiography was performed using a $12.5-\mathrm{mHz}$ linear-array probe specially designed for cardiac ultrasonic studies in rat models, with an HP sonos 7500 Imaging System (Philips (China) Investment Co. Ltd., Beijing, China). LV end-diastolic and end-systolic dimensions (EDD and ESD, respectively) were measured from the short-axis view of the LV at the midpapillary level. The LV FS was calculated as FS $=(\mathrm{EDD}-\mathrm{ESD}) / \mathrm{EDD} \times 100 \%$. All measurements were performed in triplicate in a blinded fashion. Furthermore, to evaluate the global cardiac contractile function, hemodynamic measurements were performed for 6 rats of each group at 4 weeks after treatment. A 2-French, high-fidelity, catheter-tipped micromanometer (model SPR-869, Millar Inc., Houston, Tex) was advanced into the ascending aorta and LV through the right carotid artery to record aorta and LV hemodynamic parameters.

\section{Statistics}

All data are presented as mean \pm standard deviation. SPSS software package for windows (version 10.0; SPSS, Chicago, Ill) was used for analysis. One-way analysis of variance test was performed in the cytotoxicity assessment. Other comparison between groups was made by Student $t$ test.

\section{RESULTS}

\section{In vitro Cytotoxicity Assessment}

The MTT assay of TA for cardiomyocytes showed that $0.05 \%$ TA had minimal cytotoxic effects (Figure 2). No statistical differences were observed between cells exposed to $0.025 \%$ and $0.05 \%(P=.053)$ solutions. To maximize the therapeutic effect of TA while avoiding its cytotoxicity, we selected $0.05 \%$ TA for the use of local injection.

\section{Surgical Mortality}

In total, 80 rats were enrolled into the present study and 13 of them died within the first 24 hours after the creation of MI. After the first echocardiographic study, another 7 rats were excluded owing to the unqualified LV function. Therefore, eventually we got 60 qualified rats for the following studies. However, 2 rats died after TA injection during the operation and no more died in the following study time. In the saline solution injection group, 1 rat died during injection and another died 2 days after injection. All rats that died were excluded from the study.

\section{MMP Enzymatic Activities, but not Expression, Was Inhibited by TA}

The inhibition of MMP activity by TA was confirmed by gelatin zymography assay. Twenty-four hours after treatment, in the lanes loaded with samples from the control group, 1 major transparent band corresponding to MMP-9 and 2 transparent bands corresponding to proMMP-2 and MMP-2 were observed. One week after treatment (day 8 after MI), the gelatinolytic activity of MMP-2/-9 declined, and only weak lytic bands were found in control group.

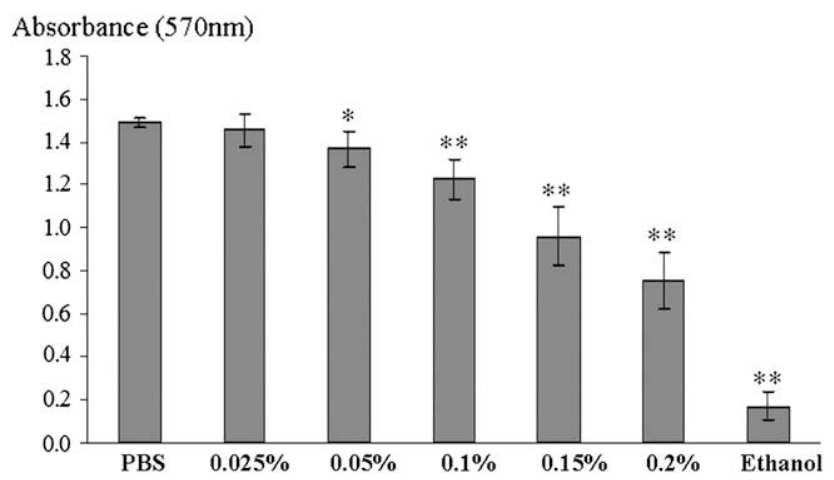

FIGURE 2. In vitro tannic acid (TA) cytotoxicity test. Cardiomyocytes were exposed to increasing concentrations of TA for 30 minutes, and then the viability was assessed by MTT assay ( $\mathrm{n}=5$ per group). Statistically significant differences $(* P<0.05$ and $* * P<0.001)$ were relative to the negative control group. $P B S$, Phosphate-buffered saline solution. 
However, in the TA treatment group, no transparent bands were observed at either of these 2 times (Figure 3, $A$ and $C$ ). These results indicate that the collagenases (MMP-2/-9) were highly activated 48 hours after MI and this activation was dramatically inhibited by TA injection and that this in vivo inhibitory effect probably could last at least 1 week. When $50 \mu \mathrm{g} / \mathrm{mL}$ TA was added to the incubation buffer, the gelatinolytic activity of MMP-2/-9 in samples from control group was also significantly inhibited (Figure 3, B), which suggests that the existence of TA can directly inhibit the enzymatic activities of MMP-2/-9.

However, there was no difference in proMMP-2, MMP2/-9, and TIMP-2 expression between TA-treated rats and saline solution-treated rats 24 hours after treatment, as indicated by Western blotting assay (Figure 3,D).

\section{Early Collagen Content Increased in TA-Treated Heart}

The effect of TA treatment on collagen network stabilization was evaluated by biochemical quantification assay. One week after treatment, the collagen content in infarcted area was significantly higher in the TA group than the control group $(48.6 \pm 7.2$ vs $37.3 \pm 6.0 \mu \mathrm{g} / \mathrm{mg}$ dry weight, $P=$ 0.027). But up to 4 weeks after treatment, the collagen content progressively increased, and the difference between the two groups vanished $(72.5 \pm 10.6$ vs $69.4 \pm 9.1 \mu \mathrm{g} / \mathrm{mg}$ dry weight, $P=0.64$ ).

\section{Evaluation of Cardiac Function}

Based on echocardiography examination at the short-axis view of the midpapillary level, both diastolic and systolic dimensions of the LV, as well as the calculated LV FS, were improved in the TA treatment group at each time point (Figure E1). On the other hand, in a hemodynamic analysis at 4 weeks after treatment, no significant difference was noted in systolic and diastolic blood pressure, as well as the maximal rising and dropping rate of LV pressure over time $( \pm \mathrm{dP} / \mathrm{dt})$ (Table E1).

\section{Cardiac Morphometry Evaluation}

As shown in Figure 4, $B$ and $D$, ECM was shown to have a more parallel and well-arranged structure in TA group than the control with the fluorescent staining. TA treatment significantly increased the scar thickness as compared with

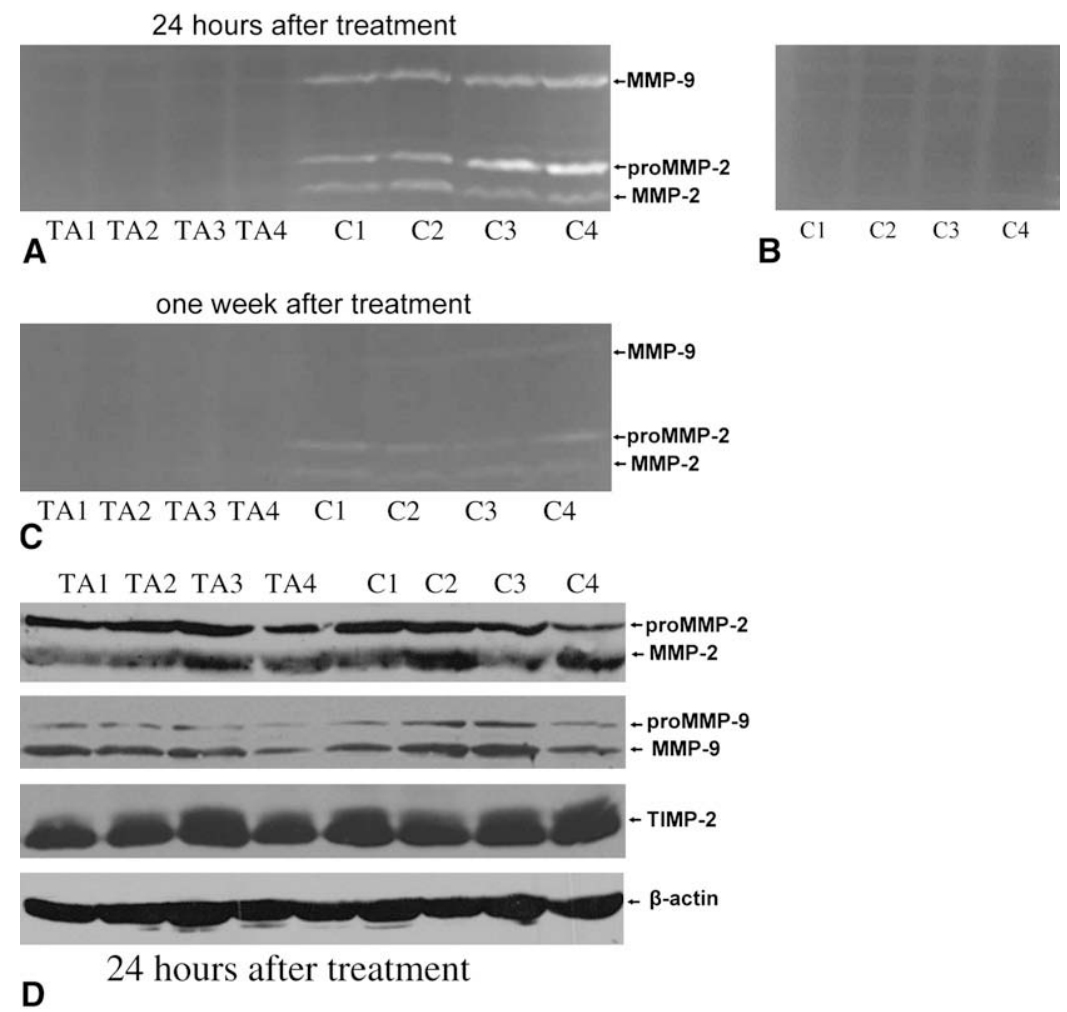

FIGURE 3. Matrix metalloproteinase (MMP)-2/-9 activity was inhibited by tannic acid (TA) treatment. $T A 1$ to $T A 4$ and $C 1$ to $C 4$ were samples from the TA group and the control group, respectively. A, Twenty-four hours after injection, gelatin zymography assay showed that MMP activity was significantly inhibited by TA treatment. B, After adding TA into the incubation buffer with a final concentration of $50 \mu \mathrm{g} / \mathrm{mL}$, the gelatinolytic activity of C1 to C4 (same samples as in Figure 2, A) was also inhibited in another gel. C, One week after injection, the MMP-2/-9 activity had declined, but the inhibitory effect of TA still remained. D, Western blotting assay for samples 24 hours after TA/saline injection, which indicated that there was no significant difference in the expression level of MMP-2, MMP-9, and their inhibitor TIMP-2. 

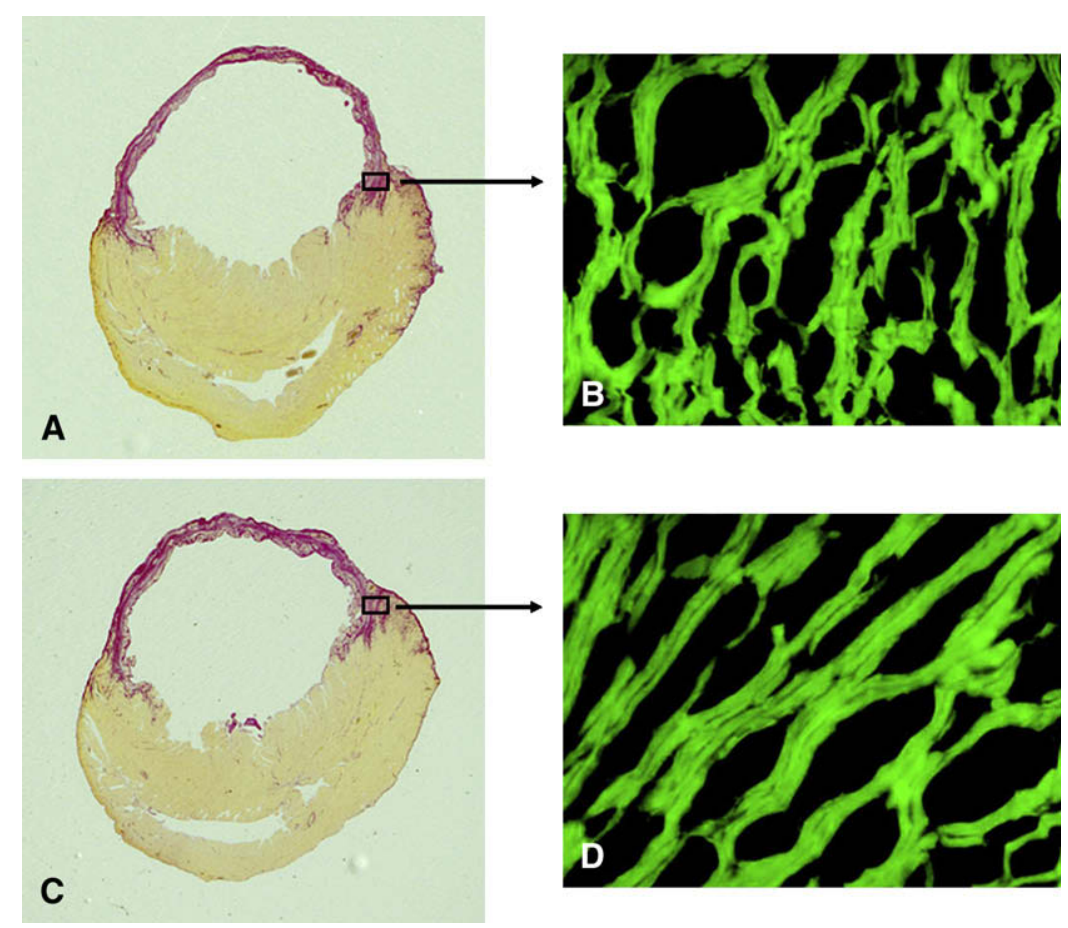

FIGURE 4. Histologic examinations of tannic acid- (TA) and saline solution-treated rats. A and C, Two representative slices stained with picrosirius red from control and TA group, respectively. Fluorescein-labeled peanut agglutinin staining demonstrated the microstructure of extracellular matrix (ECM) at the borderline zone. B, Sample from saline solution-injected rat, indicating that the ECM was arranged in a mess, and some were probably newborn. In contrast, sample D was from TA-treated rat, and the ECM fibers were mostly aligned parallel to each other and much more in order. ECM was stained green and the unstained black area represented the survival myocytes.

the saline solution injection group (Figure 4, $A$ and $C$ ). The scar length, expressed as the percentage of the total LV circumference composed of collagen, was also significantly smaller in TA treatment group than in control (Table E1). And the infarct area, also expressed as the percentage of the total LV area, was smaller in TA group. As a global marker for LV post-MI remodeling, the expansion index was also reduced after TA treatment.

\section{DISCUSSION}

In the acute phase post-MI, tissue ischemia and necrosis occur, followed by activation of MMP and enzymatic degradation of the ECM network, which causes thinning and expansion of the infarcted myocardium. Early infarct expansion has been identified as the inciting event that initiates adverse remodeling. Once established, ventricular dilation is difficult to reverse and prognosticates a poor long-term outcome. Although it is well known that the enzymatic degradation and collapse of ECM play an important role in post-MI ventricular remodeling, only few therapeutic strategies have been attempted to preserve or restore the ECM after MI.

In the present study, we chose TA injection as a novel internal cardiac support approach at 24 hours after MI to attenuate LV remodeling. The major findings of this study included: (1) TA with an appropriate concentration had a very low cytotoxicity for the cardiomyocytes; (2) intramyocardial injection of TA can increase early collagen content and inhibit MMP activity in the infarcted area early after MI; and (3) TA treatment significantly increased scar thickness, limited scar expansion, and attenuated LV dilation but did not improve the heart intrinsic contractile ability.

TA is a natural plant-derived chemical and has many beneficial actions on humans, such as anti-inflammation and antithrombosis. $^{12}$ In cardiovascular research, TA has been widely used for the fixation of bioprosthetic materials such as pericardium, heart valve, and vascular scaffold. Here, for the first time, we examined direct intramyocardial injection of TA in the treatment of MI. Compared with previous biomaterials fixation studies, the concentration of TA in the present study was dramatically reduced (from $0.3 \%$ for regular fixation purpose to $0.05 \%$ for the present study). At this concentration, TA has very low cyototoxicity on the cultured cardiomyocytes.

Based on the above in vitro cytotoxicity study, we tested the efficacy of TA injection at the acute stage post-MI. In our study, TA treatment was shown to be effective in preserving the collagen network from enzymatic degradation and consequently attenuated the scar expansion and adverse remodeling. This was verified by early collagen content assay at 1 week after injection. In previous studies, it was suggested 
that TA can cross-link collagen and elastin fibers by introducing hydrogen bonds, and thus stabilize the ECM in bioprosthetic materials fixation. ${ }^{2} \mathrm{TA}$ was thought to be able to complex or cross-link proteins by the formation of multiple hydrogen bonds and contribute to the stabilization and preservation of the dermal matrix. Isenburg and associates ${ }^{2}$ also introduced the cross-linking properties of TA into the treatment of aortic aneurysms. They reported that acute localized periadventitial delivery of noncytotoxic concentrations of pentagalloyl glucose (one derivative of TA) can inhibit elastin degeneration, attenuate aneurysmal diameter expansion, and hinder development of abdominal aortic aneurysm. In the early stage of MI, the infarcted areas remain vulnerable to expansion or even rupture until enough new ECM scaffold has been produced and matured sufficiently to resist the distending forces. The cross-linkage can protect the existing ECM from enzymatic degradation and accelerate the establishment of new collagen and elastin.

However, up to 3 weeks later, we did not observe a significant difference of collagen content. This result may be explained by the fact that in the first week after MI, the degradation of ECM outpaces its synthesis. While in the following healing stage, synthesis process is more prominent and new collagen is deposited. This alteration of degradation-synthesis balance may thus conceal the antidegradation effects of TA treatment at 4 weeks after MI. Moreover, TA is hydrosoluble and may be eluted out after a certain period, so one single-dose injection might not maintain a sustained and long-term effect on the ECM. Thus, other delivery methods, such as repeated transendocardial injection and pericardial perfusion, should be considered.

Another rationale supporting the present study is that TA may suppress MMP activity. MMPs are major contributors to the enzymatic degradation of ECM during LV remodeling. In previous tumor study, it has been demonstrated that green tea polyphenol caused strong inhibition of MMP-2, -9 , and $-12 .{ }^{4}$ Furthermore, it also was suggested that gallate residue was the most important component of the polyphenol and related to its inhibitory effect. TA contains abundant gallate residue and thus can interact with MMP. We found TA treatment significantly inhibited MMP-2/-9 activity 24 hours after TA injection (Figure 3, $A$ ), and this inhibitory effect was maintained for at least 1 week (Figure 3,C). Because of the probable astringent and anti-inflammation effects of TA, it was thought that TA treatment may inhibit inflammation and consequently inhibit the expression of MMP. Thus we also evaluated the protein level of MMP2/-9 and their inherent inhibitor TIMP-2 in the infarcted myocardium. However, no significant difference was found between the rats with or without TA treatment (Figure 3,D). Although we failed to find evidence that TA could influence the expression of MMP, we confirmed that TA can directly inhibit the total proteolytic activity of MMP in another gelatin zymography assay. When we supplemented TA in the incubation buffer (the final concentration was $50 \mu \mathrm{g} / \mathrm{mL}$, one tenth of the concentration of the TA used for in vivo injection), the gelatinolytic activity of proMMP-2, MMP-2, and MMP-9 in samples from control group was also inhibited (Figure 3, B). Therefore, we concluded that after TA local injection, the direct enzyme inhibition effect, together with collagen cross-linking, may synergistically contribute to preserve and stabilize the ECM structure.

Echocardiography and postmortem morphometry tests suggested that TA treatment was beneficial in retarding LV dilation and functional deterioration (Figure E1). But unfortunately, it seemed that TA treatment did not improve systolic and diastolic blood pressure and positive and negative $\mathrm{dP} / \mathrm{dt}$, as showed in hemodynamics examination (Table E1). Compared with stem cell therapy and therapeutic angiogenesis, TA treatment did not increase the myocardial perfusion and supply fresh new myogenic cells to restore the myocardium. Hence, it would be comprehensible that TA treatment cannot improve the heart intrinsic contractile ability, although it can maintain the integrity of ECM and attenuate the adverse remodeling. Our results are consistent with those reported by researchers who injected exogenous collagen directly into MI area. Dai and associates ${ }^{13}$ found that collagen injection did not affect the LV contractile ability, although it thickened the scar and improved the LV stroke volume and ejection fraction. Therefore, if the TA treatment is translated into clinical practice, it is expected to be considered as a supplemental therapy to maintain the integrity of ECM, and additional revascularization procedures should be performed concomitantly to improve the contractile ability of cardiomyocytes.

We also tested the body weight, liver enzyme, and creatinine level (data not shown), and we did not find any abnormality in TA group as compared with the controls. Local $0.05 \%$ TA intramyocardial injection should result in no obvious systemic toxicity. Therefore, the current study presents a safe and novel therapeutic strategy for the management of acute MI. MMP activation and ECM degradation can occur within minutes after ischemia and peak 1 to 2 days after MI. Fang and associates ${ }^{14}$ reported that LV rupture mostly occurred at a narrow time window of 2 to 6 days after MI, when the original ECM of infarct was degraded and while the healing process was not efficient. Hence, we directly injected TA at 1 day after the creation of MI. However, further experiments are needed to determine the optimal time for TA injection. Last, a paradigm shift in the understanding of matrix biology is that the ECM not only provides the structural support for cells but also provides a dynamic microenvironment for cell signaling within the intercellular space. ${ }^{15}$ The fixation of the interstitial collagen would increase the stiffness and influence the physiologic turnover and the signaling functions of ECM. On the other hand, the effect of increased scar on the injured LV in the long term may negatively impact diastolic properties of the ventricle and may be an 
arrhythmogenic substrate. Therefore, the long-term studies in large-animal models will be required before this intervention is given clinical consideration.

In summary, we intramyocardially injected TA at the early postinfarction stage, and this preclinical study, for the first time, elucidated a new internal cardiac support approach. We found that TA injection can increase the early collagen content of infarct, thicken the scar, limit scar expansion, and attenuate $\mathrm{LV}$ remodeling but did not improve the intrinsic contractile ability. This study offered a promising approach to preserve the ECM architecture after MI.

\section{References}

1. Spinale FG. Myocardial matrix remodeling and the matrix metalloproteinases: influence on cardiac form and function. Physiol Rev. 2007;87:1285-342.

2. Isenburg JC, Simionescu DT, Starcher BC, Vyavahare NR. Elastin stabilization for treatment of abdominal aortic aneurysms. Circulation. 2007;115:1729-37.

3. Isenburg JC, Simionescu DT, Vyavahare NR. Tannic acid treatment enhances biostability and reduces calcification of glutaraldehyde fixed aortic wall. Biomaterials. 2005;26:1237-45.

4. Demeule M, Brossard M, Page M, Gingras D, Beliveau R. Matrix metalloproteinase inhibition by green tea catechins. Biochim Biophys Acta. 2000;1478:51-60.

5. Li R-K, Tumiati LC, Weisel RD, Mickle DAG. Isolation of cardiomyocytes from human myocardium for primary cell culturing. Methods Cell Sci. 1993;15:147-54.
6. Labieniec M, Gabryelak T. Effects of tannins on Chinese hamster cells line B14. Mutat Res. 2003;539:127-35.

7. Hou M, Yang KM, Zhang H, Zhu WQ, Duan FJ, Wang H, et al. Transplantation of mesenchymal stem cells from human bone marrow improves damaged heart function in rats. Int J Cardiol. 2007;115:220-8.

8. Deten A, Holzl A, Leicht M, Barth W, Zimmer HG. Changes in extracellular matrix and in transforming growth factor beta isoforms after coronary artery ligation in rats. J Mol Cell Cardiol. 2001;33:1191-207.

9. Stegemann H, Stalder K. Determination of hydroxyproline. Clin Chim Acta. 1967;18:267-73.

10. Hochman JS, Choo H. Limitation of myocardial infarct expansion by reperfusion independent of myocardial salvage. Circulation. 1987;75:299-306.

11. Rhaleb NE, Peng H, Yang XP, Liu YH, Mehta D, Ezan E, et al. Long-term effect of N-acetyl-seryl-aspartyl-lysyl-proline on left ventricular collagen deposition in rats with 2-kidney, 1-clip hypertension. Circulation. 2001;103: 3136-41.

12. Freedman JE, Parker C 3rd, Li L, Perlman JA, Frei B, Zvanov V, et al. Select flavonoids and whole juice from purple grapes inhibit platelet function and enhance nitric oxide release. Circulation. 2001;103:2792-8.

13. Dai W, Wold LE, Dow JS, Kloner RA. Thickening of the infarcted wall by collagen injection improves left ventricular function in rats: a novel approach to preserve cardiac function after myocardial infarction. J Am Coll Cardiol. 2005;46: 714-9.

14. Fang L, Gao XM, Moore XL, Kiriazis H, Su Y, Ming Z, et al. Differences in inflammation, MMP activation and collagen damage account for gender difference in murine cardiac rupture following myocardial infarction. J Mol Cell Cardiol. 2007;43:535-44.

15. Fedak PW, Verma S, Weisel RD, Skrtic M, Li RK. Cardiac remodeling and failure: from molecules to man (Part III). Cardiovasc Pathol. 2005;14:109-19. 


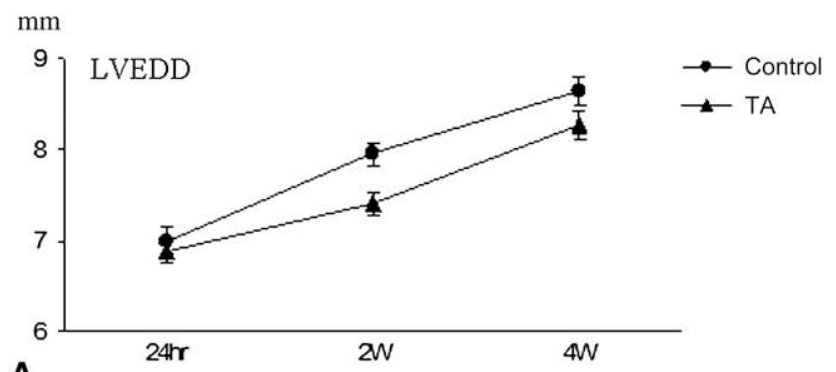

A
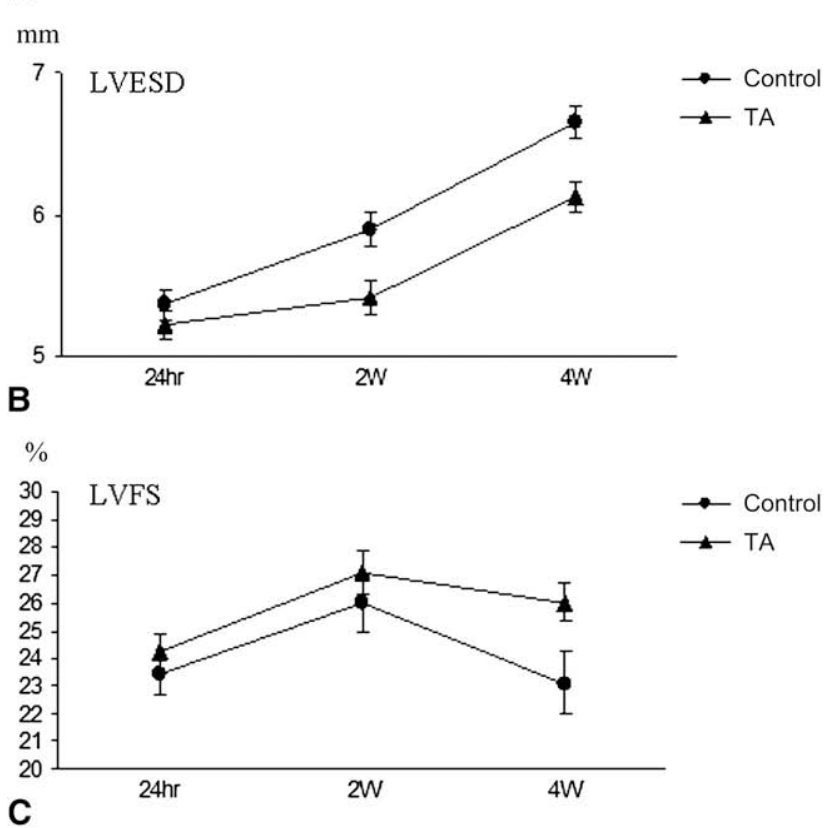

FIGURE E1. Evaluation of left ventricular dimension and function by echocardiography. There was no significant difference in the EDD, ESD, and FS between the 2 groups at the baseline. At 2 and 4 weeks after treatment, all 3 parameters in the TA group improved significantly compared with the control group $(* P<0.05, * * P<0.001) . L V E D D$, Left ventricular end-diastolic dimension; LVESD, left ventricular end-systolic dimension; $L V F S$, left ventricular fraction of shortening. 
TABLE E1. Parameters of left ventricular structure and function 4 weeks after treatment

\section{TA group}

$$
\begin{aligned}
93.8 & \pm 8.2 \\
70.6 & \pm 6.9 \\
3,879 & \pm 213 \\
1,407 & \pm 316 \\
& \\
764 & \pm 63 \\
16.0 & \pm 2.3 \\
46.6 & \pm 7.5 \\
1.04 & \pm 0.15
\end{aligned}
$$

$-\mathrm{dP} / \mathrm{dt}$

Postmortem morphometry

Scar thickness $(\mu \mathrm{m})$

Infarcted area $(\%)$

LV circumference of scar $(\%)$

Infarct expansion index
Saline solution group

$P$ value

\begin{tabular}{cc}
$90.6 \pm 8.5$ & .56 \\
$73.1 \pm 8.2$ & .61 \\
$3,855 \pm 173$ & .85 \\
$1,543 \pm 156$ & .41 \\
& \\
$485 \pm 36$ & $<.001$ \\
$20.3 \pm 2.7$ & $<.001$ \\
$65.7 \pm 8.9$ & .026 \\
$1.42 \pm 0.21$ & .010 \\
\hline
\end{tabular}

$+d P / d t$, Rate of left ventricular pressure rise; $-d P / d t$, rate of left ventricular pressure drop; $T A$, tannic acid; $L V$, left ventricular. 